\title{
The changing pattern of glaucoma referrals by optometrists
}

\begin{abstract}
Purpose To identify any change in optometric referral patterns for suspect glaucoma over a 5 year period.

Method A restrospective analysis was carried out of referrals for suspect glaucoma to a hospital glaucoma clinic from 1988 and 1993. Results There was a significant decrease in the positive referral rate with time from $56 \%$ in 1988 to $37 \%$ in $1993(p=0.02)$. Intraocular pressures (IOPs) of all patients prior to referral and at presentation to the glaucoma clinic were significantly higher in 1988 (25.1 and $25.6 \mathrm{mmHg}$ respectively) than in 1993 (22.4 and $23.0 \mathrm{mmHg}$ respectively, $p<0.0005$ ). However, the presenting IOPs of eyes with glaucoma were similar in the cohorts $(27.5$ vs $26.4 \mathrm{mmHg}$, $p=0.33$ ). Prior to referral, optometrists performed visual fields on $28 \%$ of patients in 1988 and on $48 \%$ in 1993 ( $p 0.01$ ), giving an estimate of the cup/disc ratio in $15 \%$ of patients in 1988 and $41 \%$ in 1993 ( $p=0.0004)$. Conclusions The increased false positive referral rate appears to be associated with the increasing use of visual field analysers by optometrists and a willingness to refer at lower IOPs. Increased false positive referrals result in increased waiting times for hospital assessment.
\end{abstract}

Key words Glaucoma, Optometrists, Screening

Over the last 16 years a number of authors have outlined the methods by which optometrists screen for glaucoma in the UK. ${ }^{1-5}$ In addition, data have been presented on the accuracy of optometric referrals. ${ }^{6-11}$ As over $90 \%$ of referrals for suspect chronic glaucoma are initiated by optometrists in the $\mathrm{UK}^{11}$ any major change in the methods of routine glaucoma screening utilised by optometrists might lead to increased false positive referrals if the test results were not interpreted appropriately. To date, all published studies investigating the referral practice and accuracy of optometrists have been 'snapshots in time'. Such studies do not necessarily permit the outcome of any changes in practice to be assessed. To determine whether any changes in optometric practice and referral accuracy had occurred with time in our area, a retrospective study on referrals initiated by optometrists to a glaucoma clinic was performed.

\section{Methods}

The author has supervised a consultant-led glaucoma clinic since 1986. With the use of the hospital's computerised clinic database, all new referrals to this clinic in 1988 were identified and all medical records containing a copy of the optometric referral note to the general practitioner (form GOS 18) were retained for analysis. An identical number of referrals were randomly selected from a spreadsheet of new patient attendances to the same clinic 5 years later (i.e. in 1993) in order to provide a comparable group to study.

Demographic and clinical data were extracted from the case notes and the GOS 18 forms onto study proformas and thence onto a microcomputer database for analysis.

Mann-Whitney $U$-tests and chi-squared tests were used where appropriate for statistical analysis.

For the purposes of this study, a diagnosis of 'glaucoma' was not dependent on intraocular pressure (IOP), and was made on the finding of typical excavation of the optic disc in association with nerve fibre bundle field defect(s). The field defect had to correlate with the disc appearance for the eye to be deemed to have glaucoma. The predominant method of visual field testing in 1988 in our unit was the Friedmann mark I and II instruments, whereas in 1993 most fields were performed using the 76 point screening and $24 / 2$ programmes of the Allergan Humphrey 610/614 instruments. The minimum defect on the Friedmann analyser was defined as three adjacent points recording a threshold of $0.4 \log$ units less filter than the working value. ${ }^{12}$ The minimum defect for the diagnosis of glaucoma on the Humphrey instrument was three adjacent points in a hemifield where the stimulus was only seen at least $6 \mathrm{~dB}$ brighter than expected. To compare visual field data between the two time periods, eyes with an absolute glaucomatous defect (no recorded sensitivity at a test point) extending to within $5^{\circ}$ of fixation were defined as having 'advanced disease'. 
Ocular hypertension $(\mathrm{OH})$ was defined for the purposes of this study as raised IOP $(>21 \mathrm{mmHg}$ ) measured in the clinic but no field defect on perimetry. All hospital IOPs were recorded with regularly calibrated Goldmann tonometers between 0900 and 1300 hours. Treatment is given for $\mathrm{OH}$ in our clinic when IOPs are consistently $>29 \mathrm{mmHg}$, or at a lower level when other risk factors for the development of glaucoma are present in an individual (e.g. large cup/disc ratio, cup/disc ratio asymmetry, strongly positive family history).

\section{Results}

There were 95 new patients seen in 1988 in the clinic, for 75 of which the case notes contained the GOS 18. Of the 95 randomly selected new referrals to the clinic in 1993, 71 contained the original GOS 18 . These 146 patients therefore became the study population. No significant difference was found between the ages of those at referral (mean (SD): 61.2 years (13.2) in 1988 vs 59.4 years (13.8) in 1993). Patients in 1993, however, waited longer for their outpatient assessment following optometric referral (2.4 months (2.3) vs 4.5 months (1.7), $p<0.0005)$.

\section{Hospital data}

The final diagnoses of the patients referred are given in Table 1. If glaucoma and $\mathrm{OH}$ (treated) are considered as 'positive' screens (treatable disease), then there was a statistically significant decrease in the positive referral rate over the 5 year period ( $42 / 75(56 \%)$ in 1988 vs $26 / 71$ $(37 \%)$ in 1993, $p=0.02)$.

The first IOP measured in the hospital clinic was significantly higher in the 1988 cohort (mean (SD) 25.6 $\mathrm{mmHg}(8.2)$ vs $23.0 \mathrm{mmHg}(6.5), p<0.0005)$. However, if the analysis is limited to eyes with glaucomatous field loss, there is no significant difference between the cohorts (mean IOP (SD): $27.5 \mathrm{mmHg}$ (9.6) in 1988 vs 26.4 $\mathrm{mmHg}(9.6)$ in 1993, $p=0.33)$.

Forty-six eyes (from 36 patients) had a glaucomatous visual field defect at presentation in 1988 compared with 37 eyes (from 24 patients) in 1993 ( $p=0.38$ ). Fourteen of the 46 eyes from 1988 had an advanced defect compared with 8 of the 37 eyes in $1993(p=0.51)$.

Three patients had bilateral advanced field defects in 1988 , as did 4 patients in 1993. All these patients had a presenting IOP $>21 \mathrm{mmHg}$, and in 5 of the 7 the presenting IOP was $>30 \mathrm{mmHg}$ in both eyes.

Table 1. Diagnostic categories for the two cohorts of optometric referrals

\begin{tabular}{lcr}
\hline & $1988(n=75)$ & $1993(n=71)$ \\
\hline Glaucoma & $36(48 \%)$ & $24(34 \%)$ \\
OH, treated & $6(8 \%)$ & $2(3 \%)$ \\
OH, not treated & $21(28 \%)$ & $14(20 \%)$ \\
Suspicious optic disc only & $9(12 \%)$ & $11(15 \%)$ \\
Normal & $2(3 \%)$ & $16(23 \%)$ \\
Other disease & $1(1 \%)$ & $4(5 \%)$ \\
\hline
\end{tabular}

$\mathrm{OH}$, ocular hypertension.

\section{Optometrist referral data}

The level of IOP was recorded by the optometrist in all but one case from each series. The mean (SD) IOP recorded before referral was $25.1 \mathrm{mmHg}(6.1)$ in 1988 and $22.4 \mathrm{mmHg}(5.6)$ in $1993(p<0.0005)$. The method by which the IOP was obtained was given more frequently in the later cohort $(50 / 70(71 \%)$ in 1993 vs $29 / 74(39 \%)$ in $1988, p<0.05)$, with non-contact tonometry (NCT) accounting for 42 of 50 cases in 1993 (84\%) and 22 of 29 $(76 \%)$ in 1988 . The time of day the IOP was recorded was reported equally in both cohorts $(25 / 75(33 \%)$ in 1988 vs $24 / 71(34 \%)$ in 1993).

A comment on the optic disc appearance was made in 59 of 75 cases (79\%) in 1988 and 56 of $71(79 \%)$ in 1993, but a cup/disc ratio was more frequently provided in $1993(11 / 75(15 \%)$ in 1988 vs $29 / 71$ (41\%) in 1993, $p=0.0004$ ). The true positive rate (defined as a hospital diagnosis of a glaucomatous disc with a visual field defect) for patients referred with 'pathological' discs was 8 of $36(22 \%)$ in 1988 and 8 of $24(33 \%)$ in $1993(p=0.6)$. Optometrists falsely designated one or both discs pathological in 12 of the 39 patients (31\%) with normal discs in 1988 and in 19 of 47 patients (40\%) in 1993 $(p=0.35)$.

Visual fields were performed before referral in 21 of 75 cases $(28 \%)$ in 1988 , increasing significantly to 34 of 71 $(48 \%)$ in $1993(p=0.01)$. The number of patients referred with defects that were confirmed at the hospital visit was similar in the two cohorts: 5 in 1988 and 6 in 1993.

However, 16 of the 75 patients referred with a field in 1988 were diagnosed as normal, this proportion increasing to 28 of the 71 in 1993 ( $p=0.017)$.

\section{Discussion}

Around the turn of the present decade, approximately 60000 individuals were referred to ophthalmologists every year with suspected glaucoma. ${ }^{13}$ Although it has been suggested that the number of referrals decreased following the introduction of the sight test fee in $1989,{ }^{14}$ the average interval between sight tests for patients over the age of 60 years was similar in the early 1990s and the early 1980 s. ${ }^{15,16}$

Ninety per cent of referrals now originate from optometrists. ${ }^{10,11}$ The largest study to date on optometric referral accuracy was based on 704 patients seen in 1989 and 1990 from a representative sample of UK optometrists. Glaucoma was confirmed in $40 \%$ with a further $32 \%$ requiring monitoring in the hospital setting. ${ }^{9}$ The diagnostic accuracy reported in other recent studies has varied from $32 \%{ }^{10}$ to $72 \%{ }^{8}$

This study, to our knowledge, is the first to report on two cohorts of referrals, separated in time (5 years), to the same unit. Not only can a change in diagnostic accuracy be determined, but also a change in the information provided by the optometrist to the ophthalmologist.

There was a significant reduction in the rate of true positive referrals from $48 \%$ to $34 \%$ between 1988 and 1993. Why should this have occurred? The definition of a 
positive referral in our study is considered suitable for the purposes of a two-cohort comparison, i.e. 'treatable disease' (glaucoma with field loss and $\mathrm{OH}$ resulting in treatment). We have not consciously varied the criteria for our treatment of $\mathrm{OH}$ over the 5 year period between the cohorts. Indeed, with the introduction of nerve fibre layer examination in our clinic in 1991, it is our impression that our threshold for treatment might have fallen over the 5 year period. Even when the patients treated for $\mathrm{OH}$ are excluded, the decrease in the proportion of those referred who were confirmed as having glaucoma approaches statistical significance $(p=0.08)$.

Unfortunately the numbers of glaucoma referrals to our unit as a whole and the overall true and false positive rate cannot be determined as the necessary database does not exist. It is therefore possible that there has been an increase in the numbers of individuals detected as having glaucoma per year in our area over the 5 year period.

It is interesting that we have demonstrated that the reduction in the rate of patients requiring treatment for glaucoma is associated with a reduction in presenting IOP, as measured by the optometrists and at the hospital. However, the presenting IOPs in eyes with glaucoma did not vary between the two cohorts. We can therefore conclude that optometrists were referring 'false positives' at lower IOPs in 1993 than in 1988. Could this be due to a greater tendency to refer on disc appearance in an attempt to detect more cases of lower tension glaucoma? There was a significant increase in information volunteered by optometrists on the appearance of the optic discs with time (cup/disc ratios), but no significant change in the accuracy of their assessment as determined by true and false positive rates.

Of particular interest is the significant increase in both the number of referrals who had visual field testing before referral and the number of patients referred with visual fields who were found to be negative for glaucoma at the hospital visit. Whilst it would be inappropriate to consider that the increase in false positive referrals was a direct result of increased field testing, it is likely that field testing plays a significant role.

Many optometrists arrange for visual fields to be performed routinely prior to their examination of their client. A finding of a 'positive test', even if only a minor abnormality, may influence the assessment of the optic disc and result in a 'two-hit' referral. It is therefore suggested that an optometrist should make an assessment of the optic disc before viewing the field data. This approach has been used in our clinic for some years and has been found to be helpful in the training of both ophthalmologists and optometrists in disc assessment. When a field does not correlate with a disc, a careful search for other causes of the defect should be made, including false positives such as ptosis or blepharochalasis, and 'true positives' such as retinal pigment epithelial atrophy.
The College of Optometrists has recently published guidelines for its members on screening for glaucoma. The College advises that all individuals undergoing a sight test over the age of 40 years should have their visual fields performed. ${ }^{17}$ This particular guideline may have been instituted as a result of the finding that optometrists who performed field tests in addition to tonometry in the late 1980s referred more cases of glaucoma. ${ }^{9}$ We have previously demonstrated that routine field testing in a population of individuals over the age of 49 years by trained staff using a careful protocol with a semi-automated analyser (similar to many used by optometrists) results in the discovery of 10 patients with field defects from other causes for each patient with a glaucomatous defect. ${ }^{18}$ These data and those of the present study suggest that, in order to estimate the effect of their implementation, it may have been wiser for the College of Optometrists to have piloted their guidelines in a formal manner before publishing them nationwide. Whilst there is little doubt that the extra information made available by a carefully performed field is of value in the case of a suspicious disc or in markedly raised IOP, it must be interpreted carefully before a decision is made to refer patients to an ophthalmologist. This is particularly so when they appear to have normal discs and IOPs below $24 \mathrm{mmHg}$.

In 1993 , approximately $50 \%$ of referrals to our glaucoma clinic initiated by an optometrist included a visual field assessment. If the College of Optometrists guidelines are followed, this should soon approximate to 100\%. Between 1988 and 1993 the waiting time for a routine appointment in the clinic had almost doubled. Although the inclusion of a field aids the ophthalmologist in the process of prioritisation when allocating appointments, the additional waiting time made necessary by increased false positive referrals will create much anxiety in those referred, whether they turn out to be positives or negatives. We have recently distributed local guidelines for the referral of glaucoma suspects by optometrists to our department. These were ratified by the members of our local optometric committee prior to their distribution. Future audit will reveal whether a change in the pattern of referrals occurs following this collaborative initiative.

\section{References}

1. Vernon SA, Henry DJ. Do optometrists screen for glaucoma? Eye 1989;3:131-4.

2. Strong NP. How optometrists screen for glaucoma. Ophthalmic Physiol Opt 1992;12:3-7.

3. Tuck MW, Crick RP. Use of visual field tests in glaucoma detection by optometrists in England and Wales. Ophthalmic Physiol Opt 1994;14:227-31.

4. Tuck MW, Crick RP. Non-contact tonometry: optometrists' current practice in England and Wales. Ophthalmic Physiol Opt 1994;14:347-50.

5. Steinmann WC. The 'who' and 'how' of detecting glaucoma. BMJ 1982;285:1091-3.

6. Clearkin L, Harcourt B. Referral pattern of true and suspected glaucoma to an adult ophthalmic outpatient clinic. Trans Ophthalmol Soc UK 1983;103:284-7. 
7. Brittain GPH, Austin DJ. A prospective survey to determine sources and diagnostic accuracy of glaucoma referrals. Health Trends 1988;20:43-4.

8. Harrison RJ, Wild JM, Hobley AJ. Referral patterns to an ophthalmic outpatient clinic by general practitioners and ophthalmic opticians and the role of these professionals in screening for ocular disease. BMJ 1988;297:1162-7.

9. Tuck MW, Crick RP. Efficacy of referral for suspected glaucoma. BMJ 1991;302:998-1000.

10. Sheldrick JH, Ng C, Austin DJ. An analysis of referral routes and diagnostic accuracy in cases of suspected glaucoma. Ophthalmic Epidemiol 1994;1:31-9.

11. Bell RWD, O'Brien C. Accuracy of referral to a glaucoma clinic. Ophthalmic Physiol Optics 1997;17:7-11.

12. Hugkulstone CE, Vernon SA. Use of a blue filter in visual field analysis. Br J Ophthalmol 1991;75:155-7.
13. Tuck MW. Referrals for suspected glaucoma: an International Glaucoma Association survey. Ophthalmic Physiol Opt 1991;11:22-6.

14. Laidlaw DAH, Bloom PA, Hughes AO, Sparrow JM, Marmion VJ. The sight test fee: effect on ophthalmology referrals and rate of glaucoma detection. BMJ 1994;309:634-6.

15. Optics at a glance. London: The Federation of Ophthalmic and Dispensing Opticians, 1995.

16. Ritchie J, Jacoby A, Bone M. Access to primary health care: an enquiry carried out on behalf of the United Kingdom health departments. London: HMSO, 1981.

17. The routine eye examination. Guideline advice on best practice. London: College of Optometrists, 1997.

18. Vernon SA, Henry DJ, Cater L, Jones SJ. Screening for glaucoma in the community by non-ophthalmically trained staff using semi-automated equipment. Eye 1990;4:88-97. 